\title{
Association between pre-intensive care unit (ICU) hospital length of stay and ICU outcomes in a resource- limited setting
}

\author{
S Khan, ${ }^{1} \mathrm{MB}$ ChB, DA (SA); R Wise, ${ }^{1,2,3} \mathrm{MB}$ ChB, MMed (Anaes), FCA (SA), Cert Critical Care (SA), DipObst (SA), DipPEC (SA) \\ S M Savarimuthu, ${ }^{4} \mathrm{MD}$; G L Anesi, ${ }^{5} \mathrm{MD}, \mathrm{MSCE}, \mathrm{MBE}$ \\ ${ }^{1}$ Discipline of Anaesthesia and Critical Care, School of Clinical Medicine, University of KwaZulu-Natal, Durban, South Africa \\ ${ }^{2}$ Intensive Care Department, John Radcliffe Hospital, Oxford University Trust Hospitals, Oxford, UK \\ ${ }^{3}$ Faculty Medicine and Pharmacy, Vrije Universiteit Brussel (VUB), Brussels, Belgium \\ ${ }^{4}$ Division of Pulmonary, Critical Care and Sleep Medicine, Yale School of Medicine, New Haven, CT, USA \\ ${ }^{5}$ Division of Pulmonary, Allergy, and Critical Care, Perelman School of Medicine, University of Pennsylvania, Philadelphia, PA, USA
}

Corresponding author: S Khan (sumayyahk89@gmail.com)

\begin{abstract}
Background. Previous studies demonstrated higher mortality for patients with a longer pre-intensive care unit (ICU) hospital length of stay (LOS), in well-resourced settings.

Objectives. The study aimed to determine the association between pre-ICU hospital LOS and ICU outcomes in a resource-limited setting. We hypothesised that longer pre-ICU hospital LOS would be associated with higher ICU mortality.

Methods. This was a retrospective cohort study measuring the association between pre-ICU hospital LOS and ICU outcomes using data extracted from a regional hospital ICU in KwaZulu-Natal, South Africa. Consecutive ICU admissions of all patients (medical and surgical) older than 18 years were included during the study period September 2014 to August 2018. A corrected sample size of 2040 patients was identified. Multivariable logistic regression was used to assess the primary outcome of ICU mortality, and multivariable Cox proportional hazard regression was used for the secondary outcome of ICU LOS.

Results. The median pre-ICU hospital LOS was 1 day (interquartile range (IQR) 0 - 2 days). The median length of ICU stay was 2.4 days (IQR 1.1 - 4.8 days) and the observed ICU mortality was 16\% ( $n=327 / 2040)$. Pre-ICU hospital LOS was not associated with ICU mortality in the unadjusted (odds ratio (OR) 1.00; 95\% confidence interval (CI) $0.98-1.02 ; p=0.68 ; n=2040$ ) and fully adjusted logistic regression models (OR 1.00; 95\% CI $0.98-1.03 ; p=0.90 ; n=1$ 981) using a complete case analysis for missing patient-level covariates. In Cox proportional hazard models, there was no association between pre-ICU hospital LOS and ICU LOS (hazard ratio 1.00; 95\% CI 0.98 - 1.03; $p=0.72 ; n=1$ 967), including when stratified by admission source.

Conclusions. Pre-ICU hospital LOS was not associated with either ICU mortality or ICU LOS in a resource-limited setting. Future studies should aim to include multicentre data and evaluate long-term outcomes.

Keywords. intensive care; length of stay; outcome; South Africa; mortality.

South Afr J Crit Care 2021:37(3):98-103. https://doi.org/10.7196/SAJCC.2021.v37i3.500
\end{abstract}

Contribution of the study. The study was conducted in a resource-limited setting and found no association between prolonged LOS pre-ICU and patient outcomes. Several potential explanations for this observation have been explored. This important subject is pertinent to the appropriate use of limited resources and encourages future studies to evaluate this association and to consider longer-term outcomes (e.g. 30-day mortality) in future findings.

The World Federation of Societies of Intensive and Critical Care Medicine define an intensive care unit (ICU) as an organised system for the provision of care to critically ill patients. These patients have greater physiological demands, requiring specialised medical and nursing care, enhanced monitoring and greater organ support measures to manage life-threatening illnesses. ${ }^{[1]}$ The timely administration of appropriate therapy is required to reverse pathological processes, preserve organ function, and improve outcomes and survival. ${ }^{[2-5]}$ Delayed admission to the ICU may result from errors in triage, delays in identification of critically ill patients, lengthy waiting periods for inter-hospital ICU transfer, prolonged pre-ICU hospital length of stay (LOS) (for a variety of reasons), and ICU bed unavailability.

Prior research has cited prolonged pre-ICU hospital LOS as an independent marker of hospital mortality. ${ }^{[3]}$ Patients admitted to ICU from hospital wards have significantly higher severity of illness scores compared with those admitted directly from the emergency department (ED) ${ }^{[4]}$ Furthermore, patients admitted to ICU from another hospital have similarly displayed a higher mortality. ${ }^{[4]}$

Several reasons have been suggested for worse outcomes in patients admitted to ICU following in-hospital ward stays, compared with direct 
ICU admissions from the ED. These include: (i) critically ill patients receiving limited/ suboptimal care in hospital wards by nonspecialised staff; (ii) missed warning signs of deterioration or missed opportunities for early interventions; (iii) slow deterioration of physiological function in hospital, leading to critical care admission hours to days later ${ }^{[2,3]}$ and (iv) ICU admission following events of cardiac arrest in hospital wards, which often resulted in an elevated mortality rate. ${ }^{[4]}$

Although no international consensus exists for defining the timeframe for ICU admissions, the Society of Critical Care Medicine (SCCM) recommends that patients be admitted to the ICU within 6 hours of the decision to admit from the ED. ${ }^{[9]}$ Multiple emergency medicine studies have concluded that prolonged ED LOS for patients requiring ICU admission is independently associated with higher risks of hospital mortality. ${ }^{[10-12]}$ A study from Brazil showed that each hour delay in hospital wards awaiting ICU transfer was associated with a $1.5 \%$ increased risk of ICU mortality and a $1 \%$ increased risk of hospital mortality. ${ }^{[2]}$ Similar results from a 2004 UK study demonstrated increased mortality in relation to increased time in hospital wards before ICU admission. ${ }^{[13]}$ Furthermore, a USA study found that critically ill patients who experienced delays of more than 6 hours in ED prior to ICU transfer had a longer hospital LOS with higher ICU and hospital mortality. ${ }^{[14,15]}$ Therefore, previous studies collectively showed a higher mortality for patients with a longer pre-ICU hospital LOS, primarily in well-resourced settings. ${ }^{[13]}$

One of the key contributors to prolonged pre-ICU LOS is limited ICU capacity, which has been demonstrated in American and European studies, but chronic shortages are far more prominent in low- and middle-income countries, with larger population-to-ICU-bed ratios and greater resource limitations. ${ }^{[6]} \mathrm{A}$ South African (SA) study published in 2015 reported a 1:32 000 ICU-beds-to-population ratio in the SA public sector, in contrast to the 1:5 000 ratio noted in well-resourced settings such as Germany and the USA. ${ }^{[7]}$ Moreover, longer pre-ICU hospital LOS has been associated with more costly ICU stays, with relevance to resource-limited environments. ${ }^{[8]}$

This study aimed to determine the association between pre-ICU hospital LOS and ICU outcomes in a resource-limited setting. We hypothesised that longer pre-ICU hospital LOS, adjusted for other patient-level factors, would be associated with higher in-ICU mortality.

\section{Methods \\ Study design and data source}

We performed a retrospective cohort study to measure the association between pre-ICU hospital LOS and ICU outcomes, using data extracted from a regional hospital ICU in KwaZulu-Natal, SA. Data were analysed from the ICU's electronic patient database, which collects detailed patient data at the time of ICU admission and discharge. ${ }^{[6]}$ This database has been used in prior observational studies involving measures of patient triage and acuity. ${ }^{[16]}$

Approval for the research was obtained from the Biomedical Research and Ethics Committee (ref. no. BREC/00000962/2020) and by the Institutional Review Board of the University of Pennsylvania (Philadelphia, USA). Additional permission was obtained from the study hospital and the Provincial Department of Health prior to commencement.

\section{Study site}

The study hospital is a 900-bed, regionallevel metropolitan hospital in KwaZuluNatal, SA, and serves a population of $\sim 1.4$ million people. The hospital has 6 adult mixed medical-surgical ICU beds and 3 high-care beds. The ICU is run as a closed unit, led by a team of intensivists and anaesthesiologists. In light of the limited bed capacity, patient selection for ICU admission is stringent and guided by SCCM classification, ${ }^{[17]}$ based on the physiological and organ support required, weighed against the reversibility of the pathology and the final prognosis.

\section{Study population}

The study included consecutive admissions of all patients older than 18 years of age, admitted to ICU for both medical and surgical indications. All patients admitted to the ICU and captured on the electronic patient database during the study period September 2014 to August 2018 were reviewed. A sample of 2119 was identified, of which 79 patients were excluded owing to missing data, making the corrected sample size 2040 patients (Fig. 1). For prespecified stratified secondary analyses, ICU admissions were categorised based on the source of referral, being the ED, operating theatre (OT), or hospital wards.

Patients were excluded from the study if younger than 18 years old and if the referral was an inter-hospital transfer. Interhospital transfers were excluded based on confounders arising from logistical and transportation delays within the provincial healthcare system. Therefore, patients included in this study were restricted to

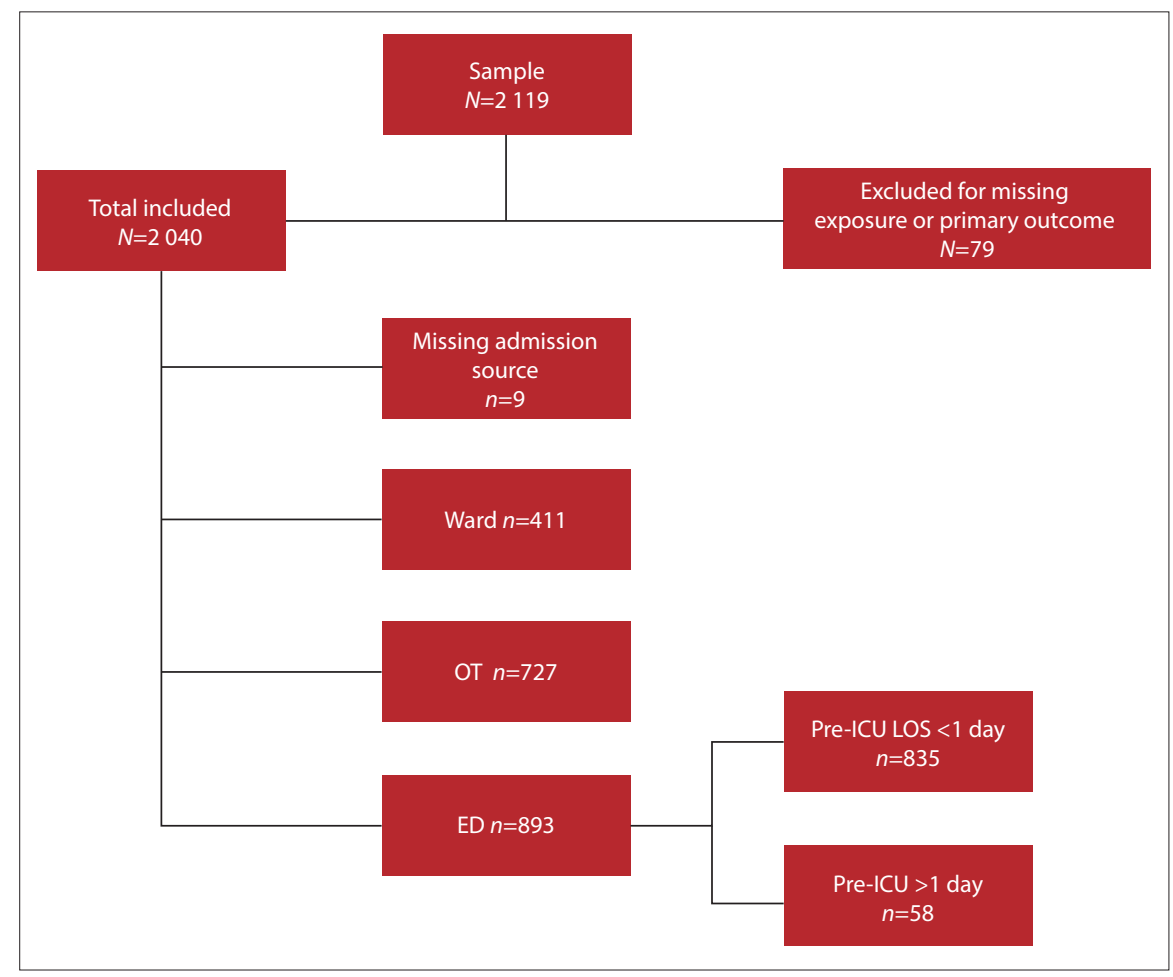

Fig.1. Flow diagram of study population sample. (OT = operating theatre; $E D=$ emergency department; $I C U=$ intensive care unit; $L O S=$ length of stay.) 
those admitted to ICU from within the study hospital's ED, OT or hospital wards.

\section{Exposures, outcomes, and adjustment variables}

The primary exposure was pre-ICU hospital LOS, which was treated as a continuous variable in calendar days for the primary analysis and for the OT and ward subgroup analyses. Among the ED subgroup, pre-ICU hospital LOS was dichotomised to $<1$ day v. $\geq 1$ day. The primary outcome was ICU mortality, defined as death in the ICU or a palliative discharge from the ICU. The secondary outcome was ICU LOS, which was measured from the time of ICU admission to the time of ICU discharge. Adjustment variables included age, gender, race, year, HIV status, chronic comorbidities, and Mortality Probability Admission Model-III $\left(\mathrm{MPM}_{0}-\mathrm{III}\right),{ }^{[18]}$ a composite model based on clinical and historical data obtained at the time of ICU admission. Comorbidities included binary present or absent indicators of: cardiovascular disease, respiratory disease, diabetes, haematological malignancy, neurological disease, HIV and highly active antiretroviral therapy (HAART) status, and nonHIV-related immunosuppression. $\mathrm{MPM}_{0}$-III includes: acute physiology indicators (heart rate $\geq 150 \mathrm{bpm}$, systolic blood pressure $\leq 90 \mathrm{mmHg}$, and Glasgow Coma Score <5); chronic diagnoses (chronic kidney disease, cirrhosis, metastatic cancer); acute diagnoses (acute kidney injury, cardiac arrhythmia, cerebrovascular accident, gastrointestinal bleeding, and intracranial mass effect); age; cardiopulmonary resuscitation attempt before ICU admission; mechanical ventilation status at ICU admission; medical or non-elective surgical admission; and resuscitation status. ${ }^{[19]}$

\section{Data analysis}

Multivariable logistic regression was used to assess the primary outcome of ICU mortality, and multivariable Cox proportional hazard regression was used for the secondary outcome of ICU LOS, with death as a censoring event. Our primary analysis included all eligible patients. We performed additional pre-specified stratified secondary analyses separately among patients admitted from the ED, OT, and ward. All analyses were conducted using STATA version 14.1 (StataCorp., USA). A $p$-value $<0.05$ was considered statistically significant.

\section{Results \\ Patient demographics}

A total of 2119 patients were admitted and entered onto the ICU database from September 2014 to August 2018. Seventy-nine patients were excluded owing to missing exposure or primary outcome variables, leaving a total sample size of 2040 patients.

The mean age of the population was 39.3 years (range 18 - 95 years), and $55.6 \%$ were male (Table 1). ICU admissions were categorised as medical or surgical, based on the referring discipline and indication for admission, of which the vast majority (73.4\%) were surgical admissions. The surgical category included admissions from general surgery, trauma surgery, obstetrics and gynaecology, orthopaedics, otorhinolaryngology, maxillofacial surgery, urology, burns and anaesthesia. Of the surgical admissions, $n=653 / 1498$ (43.6\%) had trauma as the primary reason for ICU admission. These findings are in keeping with a previous study conducted in 2015, which assessed referral patterns to this ICU. ${ }^{[7]}$

\section{Comorbidity status/illness profile}

Pre-existing comorbidities were considered, based on the primary organ system affected as depicted in Table 1. Cardiovascular comorbidities included conditions such as hypertension and ischaemic heart disease, and were present in $14.0 \%(n=285 / 2040)$ of admissions. Respiratory illnesses included asthma, chronic obstructive pulmonary disease and pulmonary tuberculosis, while pre-existing neurological disease encompassed conditions such as epilepsy and cerebrovascular events. Of note, diabetes mellitus was a common comorbidity reflected in $12.0 \%$ ( $n=244 / 2$ 040) of all admissions.

HIV was noted as the most prominent comorbidity in the study population. Patients who at presentation were known to be HIV-positive accounted for $23.4 \%$ ( $n=477 / 2$ 040) of all ICU admissions, of whom $77.8 \%(n=371 / 477)$ were on HAART. This represents a marginally higher prevalence rate than the estimated $19 \%$ reported for HIV in the general adult population of SA according to Statistics South Africa 2018. ${ }^{[20]}$ However, the figure quoted in our study may underestimate the burden of HIV in this critically ill population, as it recognises only patients with a known HIV status from medical history. HIV testing on admission is not routine practice in the ICU and is only performed if relevant to diagnostic testing and treatment.

\section{ICU acuity}

By $\mathrm{MPM}_{0}$-III, study patients had a mean predicted mortality of $18.3 \%$, which compares closely with international data on ICU mortality

\begin{tabular}{|c|c|}
\hline & $n(\%)^{*}$ \\
\hline \multicolumn{2}{|l|}{ Gender } \\
\hline Male & $1135(55.6)$ \\
\hline Female & $904(44.3)$ \\
\hline \multicolumn{2}{|l|}{ Admission type } \\
\hline Not specified & $51(2.5)$ \\
\hline Medical & $491(24.1)$ \\
\hline Surgical & $1498(73.4)$ \\
\hline Trauma & $653(43.6)$ \\
\hline Non-trauma & $845(56.4)$ \\
\hline \multicolumn{2}{|l|}{ Comorbidities } \\
\hline \multicolumn{2}{|l|}{ HIV } \\
\hline Negative or status unknown & $1563(76.6)$ \\
\hline Positive & $477(23.4)$ \\
\hline Positive, on HAART & $371(18.2)$ \\
\hline Positive, not on HAART & $106(5.2)$ \\
\hline Cardiac & $285(14.0)$ \\
\hline Diabetes mellitus & $244(12.0)$ \\
\hline Respiratory & $75(3.7)$ \\
\hline Neurological & $45(2.2)$ \\
\hline Immunosuppressed & $5(0.3)$ \\
\hline Haematological & $1(0.05)$ \\
\hline \multicolumn{2}{|l|}{ ICU severity scoring } \\
\hline \multicolumn{2}{|l|}{ qSOFA score } \\
\hline $0-1$ & $783(38.4)$ \\
\hline 2 & $869(42.6)$ \\
\hline 3 & $367(18.0)$ \\
\hline Missing & $21(1.0)$ \\
\hline \multicolumn{2}{|l|}{$\mathrm{MPM}_{0}-\mathrm{III}, \%$} \\
\hline 25th percentile & 3.2 \\
\hline 50th percentile & 13.4 \\
\hline 75th percentile & 26.6 \\
\hline 95th percentile & 57.3 \\
\hline Mean (SD), \% & $18.3(18.2)$ \\
\hline Pre-ICU LOS (days), mean (IQR) & $1(0-2)$ \\
\hline \multicolumn{2}{|c|}{$\begin{array}{l}\text { HAART = highly active antiretroviral therapy; } \mathrm{qSOFA}=\text { Quick Sequential Organ } \\
\text { Failure Assessment; } \mathrm{MPM} \mathrm{M}_{0} \mathrm{-III}=\text { Mortality Probability Assessment Model-III; } \\
\mathrm{SD}=\text { standard deviation; } \mathrm{IQR}=\text { interquartile range. } \\
{ }^{*} \text { Unless otherwise specified. }\end{array}$} \\
\hline
\end{tabular}


rates. ${ }^{[21]}$ Over $60 \%$ of the study population was found to have a Quick Sequential Organ Failure Assessment (qSOFA) score $\geq 2$, which is associated with an increased risk of hospital mortality and a prolonged ICU stay defined as ICU LOS $\geq 3$ days. ${ }^{[16,22]}$

\section{Outcomes}

The majority of patients, $43.8 \%(n=893 / 2040)$, were admitted to ICU from the ED. The pre-ICU LOS for patients admitted from ED corresponded with ED LOS, which reflected that 93.5\% ( $n=835 / 893)$ of patients were admitted to ICU within 24 hours. Further admissions from OT and the hospital wards accounted for $35.6 \%(n=727 / 2040)$ and $20.2 \%(n=411 / 2040)$, respectively (Table 2$)$.

The median pre-ICU hospital LOS was 1 day (interquartile range (IQR) 0 - 2 days). The median length of ICU stay was 2.4 days (IQR $1.1-4.8$ days) and the observed ICU mortality was $16.0 \%$ ( $n=327 / 2$ 040) (Table 3).

Pre-ICU hospital LOS was not associated with ICU mortality in the unadjusted (odds ratio (OR) 1.00, 95\% confidence interval (CI) $0.98-1.02 ; p=0.68 ; n=2040)$ and fully adjusted logistic regression models (OR 1.00; 95\% CI $0.98-1.03 ; p=0.90 ; n=1981$ using a complete case analysis for missing patient-level covariates). In analyses stratified by admission source, pre-ICU hospital LOS was likewise not associated with ICU mortality for patients admitted from the OT (OR 1.00, 95\% CI 0.97 - 1.04; $p=0.79 ; n=702$ ), ED (OR 0.90; 95\% CI 0.60 - 1.35; $p=0.61$; $n=871$ ), or ward (OR 1.02; 95\% CI $0.97-1.07 ; p=0.48 ; n=394$ ) (Table 3).

\begin{tabular}{ll}
\multicolumn{2}{l}{ Table 2. Pre-ICU source of admission } \\
\hline Source of ICU referral & $\boldsymbol{n}(\mathbf{0 4 0})$ \\
\hline ED & $893(43.8)$ \\
$\leq 1$ day & $835(93.5)$ \\
$>1$ day & $58(6.5)$ \\
OT & $727(35.6)$ \\
Wards & $411(20.2)$ \\
Missing data & $9(0.4)$ \\
ICU = intensive care unit; $\mathrm{ED}=$ emergency department; OT = operating theatre.
\end{tabular}

In Cox proportional hazard models, there was no association between pre-ICU hospital LOS and ICU LOS (hazard ratio 1.00; 95\% CI $0.98-1.03 ; p=0.72 ; n=1967)$, a secondary outcome, including when stratified by admission source (Table 3 ).

\section{Discussion}

In contrast to previous studies from middle- and high-income countries, pre-ICU hospital LOS was not associated with ICU LOS or ICU mortality based in a resource-limited setting. This may be attributed to a number of patient and institutional factors associated with the study population, hospital, and this particular resource-constrained environment.

International data were analysed in the ICON (Intensive Care Over Nations) audit published in 2014, which assessed the worldwide burden of critical illness across 84 countries. ${ }^{[21]}$ The worldwide statistics for ICU mortality were noted to be $16.2 \%$, with a median ICU LOS of 3 days. The audit included region-specific statistics with African figures for ICU mortality of $16.9 \%$ and median ICU LOS of 2 days. The audit was skewed in its representation, with Europe comprising $>50 \%$ of the study population and Africa representing $1.4 \%$. These results may better reflect European data and should be interpreted with caution when considering Africa. Although there is a paucity of data from African countries regarding in-ICU mortality, our study reported an ICU mortality of $16 \%$ and a median ICU LOS of 2.4 days, which parallel international statistics.

Our study population was younger (median age of 35 years) compared with previous studies (median age 50 - 60 years). ${ }^{[2,3]}$ Moreover, the study population had less chronic burden of disease (37\%), which is likely to influence mortality. In addition, more than $70 \%$ of the study population was admitted for surgical rather than medical indications, and of these patients $>40 \%$ were admitted on the basis of severe acute traumatic injuries. This differs from studies in high-income countries in which chronic disease rates are greater and more patients are admitted with primary medical illnesses and possibly worse baseline physiology. These differences may have a marked impact on patient survival.

Although the study population was noted to have a high burden of HIV, more than $70 \%$ of these patients were on HAART. The South African

Table 3. Association of pre-ICU hospital LOS and ICU outcomes

\begin{tabular}{|c|c|c|c|}
\hline ICU LOS (days), mean (IQR) & $2.4(1.1-4.8)$ & & \\
\hline \multicolumn{4}{|l|}{ Observed outcomes } \\
\hline \multicolumn{4}{|l|}{ ICU mortality, $n(\%)$} \\
\hline Alive & $1713(84.0)$ & & \\
\hline Dead & $327(16.0)$ & & \\
\hline ICU mortality & OR & $95 \% \mathrm{CI}$ & $p$-value \\
\hline Unadjusted mortality model & 1.00 & $0.98-1.02$ & 0.70 \\
\hline Fully adjusted mortality model & 1.00 & $0.98-1.03$ & 0.90 \\
\hline \multicolumn{4}{|l|}{ Stratified mortality analysis } \\
\hline Patients admitted from OT & 1.00 & $0.97-1.04$ & 0.79 \\
\hline Patients admitted from ED & 0.90 & $0.60-1.35$ & 0.61 \\
\hline Patients admitted from wards & 1.02 & $0.97-1.07$ & 0.48 \\
\hline ICU LOS & HR & $95 \%$ CI & $p$-value \\
\hline Unadjusted univariate LOS analysis & 1.00 & $0.98-1.02$ & 0.70 \\
\hline Fully adjusted LOS model & 1.00 & $0.98-1.03$ & 0.72 \\
\hline \multicolumn{4}{|l|}{ Stratified LOS analysis } \\
\hline Patients admitted from OT & 1.01 & $0.98-1.04$ & 0.50 \\
\hline Patients admitted from ED & 0.80 & $0.57-1.13$ & 0.21 \\
\hline Patients admitted from wards & 1.02 & $0.97-1.06$ & 0.46 \\
\hline
\end{tabular}

$\mathrm{ICU}=$ intensive care unit; $\mathrm{IQR}=$ interquartile range; $\mathrm{LOS}=$ length of stay; $\mathrm{SD}=$ standard deviation; $\mathrm{OR}=$ odds ratio; $\mathrm{CI}=$ confidence interval; $\mathrm{HR}=$ hazard ratio; $\mathrm{ED}=$ emergency department; $\mathrm{OT}=$ operating theatre. 
Surgical Outcomes Study (SASOS) conducted in 2011 noted that despite the high prevalence of HIV in the SA population, HIV infection was not associated with increased in-hospital mortality, and this population may not have a significantly increased risk of mortality based on this comorbidity alone. ${ }^{[23,24]}$

Previous studies in developed, well-resourced countries varied with regard to pre-ICU placement of patients. This may impact on these study outcomes, as patients in the ED have access to constant surveillance and doctors present in attendance when compared with wards, which may be ill-equipped, with non-specialised staff inexperienced in managing critically ill patients. In turn, benefit may be attributed to the similarity between OT and ICU environments, with care by specialised staff, advanced monitoring and necessary skills available.

However, it is interesting to note that comparable studies conducted in the UK, Portugal, and Australia also demonstrated no significant adverse outcomes on ICU LOS or mortality in association with delayed ICU admissions from ED. ${ }^{[25-27]}$ These studies classified timely admissions as being between 4 and 8 hours, and looked at patients admitted exclusively from the ED. These studies further depict the vast contrast in definitions used by resource-rich and resource-poor environments when classifying pre-ICU LOS and delays in ICU admission.

Moreover, a study conducted at the Royal London Hospital concluded that pre-ICU length of hospital stay is a predictor of hospital but not ICU mortality. ${ }^{[3]}$ This highlights the impact that delays may have on long-term outcome, which our study was not able to evaluate.

In a 2019 publication by Santos et al., ${ }^{[25]}$ it was noted that critically ill patients with prolonged hospital stays prior to ICU may have worse outcomes as a consequence of delayed monitoring and treatment administration. The outreach system of the ICU team in this study may account for improved outcomes, as patients deemed candidates for critical care admission are followed up and reviewed by the ICU team in ED and other high-fidelity areas of the hospital. The impact of such interventions may play a key role in explaining the better-than-expected outcomes demonstrated in our results. This practice may not be possible in every institution and may impact on patient triage, ICU admissions and outcomes. Furthermore, it is not uncommon for these outlying highfidelity patients to progress from ICU candidates to no longer requiring ICU, either by improvement or deterioration in clinical condition. Thus, it may be possible that a healthier, more resilient subgroup of patients ultimately fulfil the criteria for ICU admission and are therefore less likely to deteriorate rapidly.

International studies have described comparable practices of informal ICU care being implemented in temporary areas across hospitals, when faced with ICU bed shortages. In the USA, this often involves critical care delivered temporarily outside ICUs or within novel 'ED long-term acute-care facilities' which house patients that await hospital beds for longer than 6 hours. ${ }^{[15,28]}$ Similarly, a UK study described the concept of 'critical care without walls' as one that is becoming increasingly accepted, and cites the hospital's theatre suite being used to board patients pending ICU admission. In this interim period, patients are managed according to ICU specialist input and all required treatments and interventions are instigated in this setting. This practice may explain why such delayed ICU admissions did not translate to prolonged ICU stays or adverse outcomes and under certain conditions may prove beneficial to ICU outcomes. ${ }^{[2]}$

The Critical Care Society of Southern Africa Consensus Statement on ICU Triage and Rationing posed a similar question in its recent publication, attempting to answer "Are ICU "substitutes" which may not provide an equivalent level of care, acceptable alternatives?' It also commented on less severely ill patients requiring lower levels of care which may be appropriate. However, for patients requiring ICU care, but where it is unavailable because of resource constraints, limited benefit may be gained from lower levels of care. Thus, the conclusion was that if ICU beds are not available, efforts should be made to provide the best care possible in settings outside of the ICU. ${ }^{[30]}$

\section{Study limitations}

The study was limited to a single centre, which may not be representative of national ICU outcomes. However, it provides a research template for multicentre evaluation in similar resource-limited environments. $\mathrm{MPM}_{0}$-III was used as the predictive scoring tool over the more commonly used APACHE II (Acute Physiological Assessment and Chronic Health Evaluation) score owing to missing data for calculation of APACHE II ( $>30 \%)$ when compared with the more acceptable missing rate for the $\mathrm{MPM}_{0}$-III score (8.2\%). Mortality outcomes were restricted to in-ICU stay and long-term outcomes were not explored because of data unavailability. Future studies should aim to evaluate hospital mortality and 30-day survival as this may more accurately reflect the impact of delays to ICU admission. This study also differentiated admissions to ICU based on source of referral and assessed pre-ICU LOS for each. Furthermore, the time frame used for evaluating pre-ICU LOS in our study was in calendar days, whereas previous studies have looked at LOS in hours. These broad categorisations and analyses may not accurately capture distinct subgroups, and future studies could evaluate patients within narrower constraints to better assess heterogeneous relationships.

\section{Conclusions}

In contrast to previous studies, pre-ICU hospital LOS was not associated with either ICU mortality or ICU LOS in a resource-limited setting. Future studies should aim to include multicentre data and evaluate long-term outcomes.

\section{Declaration. None.}

Acknowledgements. The authors acknowledge Dr Nikki Allorto for her work in design and maintenance of the intensive care electronic database (ICED) used in this study, and Dr Carel Cairns for his prior work on data captured on the ICED system.

Author contributions. SK and RW contributed to study design, acquisition of data, interpretation of data, writing and revision of the manuscript. SMS and GLA contributed to the study design, data analysis, interpretation of data and revision of the manuscript.

Funding. This project was supported by grant number K12HS026372 (GLA) from the US Agency for Healthcare Research and Quality. The content is solely the responsibility of the authors and does not necessarily represent the official views of the Agency for Healthcare Research and Quality.

Conflicts of interest. None.

1. Marshall JC, Bosco L, Adhikari NK, et al. What is an intensive care unit? A report of the task force of the World Federation of Societies of Intensive and Critical Care Medicine. J Crit Care 2017;37:270-276. https://doi.org/10.1016/j.jcrc.2016.07.015

2. Cardoso LTQ, Grion CMC, Matsuo T, et al. Impact of delayed admission to intensive care unit on mortality of critically ill patients: A cohort study. Crit Care 2011;15(1):R28. https://doi. org/10.1186/cc 9975

3. Flavin K, Hall D, Marshall G, Zolfaghari P. Pre-ICU length of hospital stay is a predictor of hospital but not ICU mortality. Crit Care 2015;19(1):P527. https://doi.org/10.1186\%2Fcc14607

4. Flabouris A, Jeyadoss J, Field J, Soulsby T. Direct and delayed admission to an intensive care or high dependency unit following discharge from the emergency department: Associated patient characteristics and hospital outcomes. Crit Care Resus 2012;14(3):191-197.

5. Chiavone PA, Rasslan S. Influence of time elapsed from end of emergency surgery until admission to intensive care unit, on Acute Physiology and Chronic Health Evaluation II (APACHE II) prediction and patient mortality rate. Sao Paulo Med J 2005;123:167-174. https://doi.org/10.1590/ s1516-31802005000400003 
6. Wise RW, de Vasconcellos K, Skinner D, et al. Outcomes 30 days after ICU admission: The 30DOS study. South Afr J Anaesth Analg 2017;23(6):139-144. https://doi.org/10.1080/2220118 1.2017.1402553

7. Gordon K, Allorto N, Wise R. Analysis of referrals and triage patterns in a South African metropolitan adult intensive care service. S Afr Med J 2015;105(6):491-495. https://do org/10.7196/SAMJ.9007

8. Simpson K, Williams G, Quasim T. Length of hospital stay prior to ICU admission and outcome. Crit Care 2011;15(Suppl 1):P465-P. https://doi.org/10.1186\%2Fcc9885

9. Nates JL, Nunnally M, Kleinpell R, et al. ICU admission, discharge, and triage guidelines: A framework to enhance clinical operations, development of institutional policies, and further research. Crit Care Med 2016;44(8):1553-1602. https://doi.org/10.1097/ccm.0000000000001856

10. Zhang Z, Bokhari F, Guo Y, Goyal H. Prolonged length of stay in the emergency department and increased risk of hospital mortality in patients with sepsis requiring ICU admission. Emerg Med J 2019;36(2):82-87. https://doi.org/10.1136/emermed-2018-208032

11. Singer AJ, Thode HC Jr, Viccellio P, Pines JM. The association between length of emergency department boarding and mortality. Acad Emerg Med 2011;18(12):1324-1329. https://doi org/10.1111/j.1553-2712.2011.01236.x

12. García-Gigorro R, de la Cruz Vigo F, Andrés-Esteban EM, et al. Impact on patient outcome of emergency department length of stay prior to ICU admission. Med Intensiva (English Edition). 2017;41(4):201-208. https://doi.org/10.1016/j.medin.2016.05.008

13. Goldhill DR, McNarry AF, Hadjianastassiou VG, Tekkis PP. The longer patients are in hospital before intensive care admission the higher their mortality. Intensive Care Med 2004;30(10):19081913. https://doi.org/10.1007/s00134-004-2386-2

14. Gunnerson KJ, Bassin BS, Havey RA, et al. Association of an emergency department-based intensive care unit with survival and inpatient intensive care unit admissions. JAMA Network Open 2019;2(7):e197584-e. https://doi.org/10.1001/jamanetworkopen.2019.7584

15. Anesi GL, Chelluri J, Qasim ZA, Chowdhury M, Kohn R. Association of an emergency department-embedded critical care unit with hospital outcomes and intensive care unit use. Annals ATS 2020;17(12):1599-1609. https://doi.org/10.1513/annalsats.201912-912oc

16. Savarimuthu SM, Cairns C, Allorto NL, et al. qSOFA as a predictor of ICU outcomes in a resource-limited setting in KwaZulu-Natal Province, South Africa. South Afr J Crit Care 2020;36(2):92-95. http://dx.doi.org/10.7196/SAJCC.2020.v36i2.433

17. Task Force of the American College of Critical Care Medicine, Society of Critical Care Medicine. Guidelines for intensive care unit admission, discharge, and triage. Crit Care Med 1999;27(3):633-638.

18. Higgins TL, Teres D, Copes WS, Nathanson BH, Stark M, Kramer AA. Assessing contemporary intensive care unit outcome: An updated Mortality Probability Admission Model (MPM0-III). Crit Care Med 2007:35(3):827-835. https://doi.org/10.1097/01.ccm.0000257337.63529.9f
19. Anesi GL, Gabler NB, Allorto NL, et al. Intensive care unit capacity strain and outcomes of critical illness in a resource-limited setting: A 2-hospital study in South Africa. J Intensive Care Med 2020;35(10):1104-1111. https://doi.org/10.1177/0885066618815804

20. Statistics South Africa. Mid-Year Population Estimates. Pretoria: Stats SA, 2018:26.

21. Vincent J-L, Marshall J, Namendys-Silva S, et al. Assessment of the worldwide burden of critical illness: The Intensive Care Over Nations (ICON) audit. Lancet Respir Med 2014;2(5):380-386. https://doi.org/10.1016/s2213-2600(14)70061-x

22. Canet E, Taylor DM, Khor R, Krishnan V, Bellomo R. qSOFA as predictor of mortality and prolonged ICU admission in emergency department patients with suspected infection. J Crit Care 2018;48:118-123. https://doi.org/10.1016/j.jcrc.2018.08.022

23. Balkema CA, Irusen EM, Taljaard JJ, Zeier MD, Koegelenberg CF. A prospective study on the outcome of human immunodeficiency virus-infected patients requiring mechanical ventilation in a high-burden setting. QJM 2015;109(1):35-40. https://doi.org/10.1093/qjmed/hcv086

24. Biccard BM, Madiba TE. The South African Surgical Outcomes Study: A 7-day prospective observational cohort study. S Afr Med J 2015;105(6):465-475. https://doi.org/10.7196/samj.9435

25. Santos FRQ, Machado MdN, Lobo SMA. Adverse outcomes of delayed intensive care unit. Rev Bras Ter Intensiva 2020;32(1):92-98. https://doi.org/10.5935\%2F0103-507X.20200014

26. Ramaiah R, Shepard B, Hopkins P, Maharaj R. Effect of delayed ICU admission on mortality and morbidity. Crit Care 2011;15(1):P458. https://doi.org/10.1186/cc9878

27. Carter AW, Pilcher D, Bailey M, Cameron P, Duke GJ, Cooper J. Is ED length of stay before ICU admission related to patient mortality? Emerg Med Australasia 2010;22(2):145-150. https://doi. org/10.1111/j.1742-6723.2010.01272.x

28. Agustin M, Price LL, Andoh-Duku A, LaCamera P. Impact of delayed admission to the intensive care unit from the emergency department upon sepsis outcomes and sepsis protocol compliance. Crit Care Res Practice 2017;2017:9616545. https://doi.org/10.1155/2017/9616545

29. O'Callaghan DJP, Jayia P, Vaughan-Huxley E, et al. An observational study to determine the effect of delayed admission to the intensive care unit on patient outcome. Crit Care 2012;16(5):R173. https://doi.org/10.1186/cc11650

30. Joynt GM, Gopalan DP, Argent AA, et al. The Critical Care Society of Southern Africa Consensus Guideline on ICU Triage and Rationing (ConICTri). S Afr Med J 2019;109(8b):630-642. https:// doi.org/10.7196/samj.2019.v109i8b.13

Accepted 10 September 2021 